\title{
The comparison of FLOTAC, FECPAK and McMaster techniques for nematode egg counts in cattle
}

\author{
Antonio Bosco', Laura Rinaldi ${ }^{*}$, Maria P. Maurelli', Vincenzo Musella², \\ Gerald C. Coles ${ }^{3}$ and Giuseppe Cringoli' \\ ${ }^{1}$ Department of Veterinary Medicine and Animal Productions, University of Naples Federico II, CREMOPAR Regione Campania, \\ Naples, Italy; ${ }^{2}$ Department of Health Sciences, University Magna Graecia of Catanzaro, Italy; ${ }^{3}$ School of Veterinary Sciences, \\ University of Bristol, Langford House, Bristol BS40 5DU, UK
}

\begin{abstract}
Three methods, FLOTAC, FECPAK and McMaster were compared for accuracy and sensitivity for counting numbers of nematode eggs in faeces of naturally infected cattle with high or low nematode egg counts. Only FLOTAC gave positive results for 12 replicates from pooled samples with low egg counts making it more sensitive than FECPAK (67\%) and McMaster (41.7\%). FLOTAC resulted in generally higher egg counts and lower coefficients of variation than the other two methods used. The reliability of FECPAK and McMaster is depended on the area under the slide counted. All three methods can be used for making decisions whether to treat but FLOTAC or Mini-FLOTAC should be used for faecal egg count reduction tests when lower egg counts are present.
\end{abstract}

\section{Keywords}

FecPak, McMaster, FLOTAC, nematode egg count, cattle

\section{Introduction}

Anthelmintic resistance is an increasing problem in nematodes of cattle in several parts of the world (Sutherland and Leathwick 2011). If the guidelines for detection of anthelmintic resistance are used (Coles et al. 1992) egg counts should be sufficiently high to ensure that meaningful results are obtained. This is depending to the sensitivity of the method used (e.g. McMaster technique may be also up to 50 eggs per gram (EPG)). However, if more sensitive techniques are used lower egg counts can be included in studies (Levecke et al. 2011, 2012). At the same time to detect levels of resistance, the methods used for egg counts should have a variance as low as possible.

The most widely used method for faecal egg counts is the McMaster technique, developed in Australia and originally used for nematode egg counts in sheep where egg counts can be relatively high (Gordon and Whitlock, 1939). The method lacks sensitivity particularly at low egg counts (Mes, 2003). FECPAK is essentially a larger version of the McMaster slide (www.fecpak.com) and it was developed in New Zealand to provide a simple on farm method of egg counting for making decisions on whether to treat or for the determi- nation of anthelmintic efficacy. Because it starts with a larger faecal aliquot $(20 \mathrm{~g})$ rather than the $3 \mathrm{~g}$ often used for McMaster, it gives much more reliable results with equine faecal samples where eggs are not evenly distributed (Presland et al. 2005). FLOTAC is a multivalent Faecal Egg Count (FEC) technique based on the centrifugal flotation of the sample and the subsequent translation of the top layer of the floating suspension (Cringoli et al. 2010). This makes counting of the sample much easier as most of the colour and debris are no longer in the light path. Due to its high accuracy and sensitivity, FLOTAC has previously been recommended for monitoring anthelmintic drug efficacy in cattle (Levecke et al. 2011, 2012).

The present study was undertaken to compare the three techniques using faeces from naturally infected cattle with low and high egg counts of gastrointestinal (GI) strongyles to determine which is the optimum technique.

\section{Materials and Methods}

Fresh faecal samples were collected from 20 cattle excreting 210 EPG (mean 8.1 EPG) (based on FLOTAC Basic Technique) 
and from 20 cattle excreting 800-1200 EPG (mean 1112 EPG) (based on FLOTAC Basic Technique) and from these individual samples, two composite samples were prepared using 5 grams per animal, one composite having a low and one having a high GI strongyle EPG level. From each pooled sample, after a good homogenization, $30 \mathrm{~g}$ were taken and homogenized in tap water $(270 \mathrm{ml}$; dilution ratio $1: 10$, total volume $300 \mathrm{ml})$. The suspension was sieved (wire mesh aperture $=250 \mathrm{~mm}$ ) to remove large debris. Thirty-six conic tubes were filled with 6 $\mathrm{ml}$ of filtered suspension to provide 12 replicates for each of the three methods. Each aliquot was centrifuged for $3 \mathrm{~min}$ at 170 $\mathrm{x} g$, the supernatant was poured off and discarded, leaving only a pellet in the bottom of tube. Then, tubes were randomly assigned to the three techniques, FLOTAC (Cringoli et al. 2010), FECPAK (www.fecpak.com) and McMaster (Ministry of Agriculture, Fisheries and Food, 1986). For the three techniques a saturated $\mathrm{NaCl}$ flotation solution (FS2; density $=1.2$ ) was used.

For the FLOTAC the aliquot was re-suspended in FS2 up to $6 \mathrm{ml}$ level and one chamber was examined. Thus, a single flotation chamber of the FLOTAC was utilized for each replicate (analytic sensitivity $=2 \mathrm{EPG}$ ). The apparatus was then centrifuged at $120 \mathrm{xg}$ for $5 \mathrm{~min}$ and translated.

For the FECPAK, the pellet was re-suspended in FS2 up to $6 \mathrm{ml}$ level and the slides were examined. For each slide, the GI strongyles counts were performed under each of four reading levels (one grid, two grids, one chamber, two chambers), re- sulting in an analytic sensitivity of 20 EPG (one grid), $10 \mathrm{EPG}$ (two grids), 7.1 EPG (one chamber), and 3.6 EPG (two chambers).

For the McMaster, the pellet was re-suspended in FS2 up to $6 \mathrm{ml} \mathrm{level} \mathrm{and} \mathrm{the} \mathrm{slides} \mathrm{were} \mathrm{examined.} \mathrm{For} \mathrm{each} \mathrm{slide,}$ the GI strongyles counts were performed under each reading level, resulting in an analytic sensitivity of 66.6 EPG (one grid), 33.3 EPG (two grids), 20 EPG (one chamber), 10 EPG (two chambers).

GI strongyle eggs were counted at X 100 magnification.

For all replicates of the composite sample with low levels of GI strongyle eggs, the arithmetic mean EPG values (derived from the 12 replicates), the standard deviation (SD), and the Coefficient of Variation (CV) were calculated for each technique and each reading level (one grid, both grids, one chamber and both chambers). The $\mathrm{CV}$ was calculated dividing the standard deviation by the arithmetic mean EPG, i.e. $\mathrm{CV}=$ (SD/mean EPG) x 100. The same statistics were calculated for the composite sample with high EPG.

\section{Statistical analysis}

The arithmetic mean EPG were calculated for each technique. Differences between techniques and reading area were analysed using one-way ANOVA with post hoc Fisher's least significant difference (Bonferroni). All statistical analyses

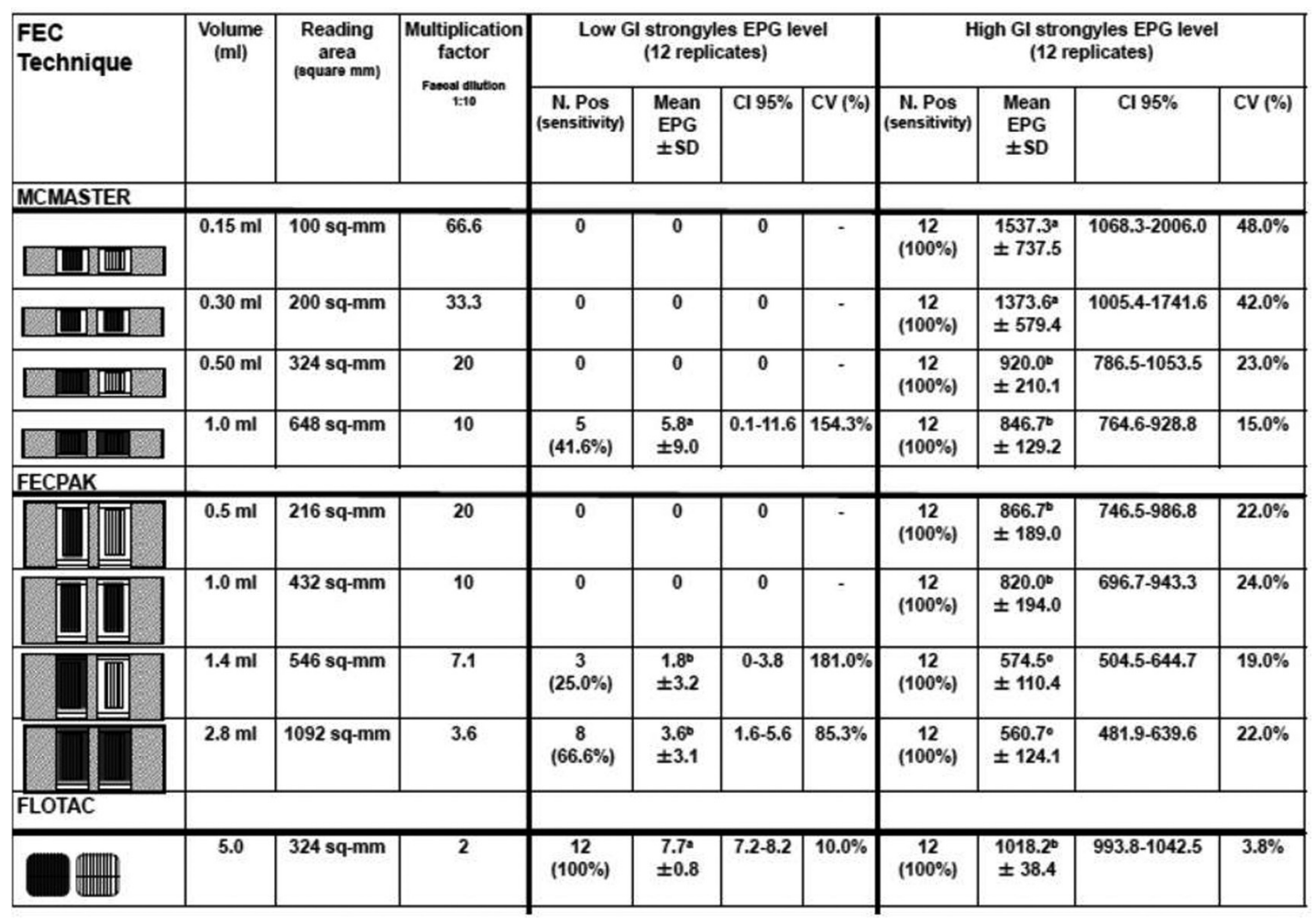

Fig.1. The comparison of FLOTAC, FECPAK, and McMaster techniques for GI strongyle faecal egg counts in cattle for composite samples with low and high burden of eggs: no. positives and sensitivity, mean EPG and accuracy $(\mathrm{CV})$ of the three methods. P $<0.05$; significant differences for different letters. 
(Mann-Whitney U-test, ANOVA) were performed using STATA 10.0 software (Stata Corp., Texas 77845, USA).

\section{Results}

Fig. 1 shows the characteristics of each reading level of each FEC technique regarding volume $(\mathrm{ml})$, reading area (square $\mathrm{mm}$ ) and multiplication factor (at the dilution ratio of 1:10) for the three apparatus that we used and the results obtained in terms of sensitivity, mean EPG, standard deviation (SD), confidence interval (CI95\%) and CV.

For composite sample with low level of GI strongyle eggs, FLOTAC always gave positive results for all replicates, thus making it more sensitive than FECPAK (66.7\%) and McMaster $(41.7 \%)$. For composite samples with high level of GI strongyle eggs, all the methods gave positive results.

FLOTAC also produced SD and CV values lower than the other two techniques, both for high and low GI strongyle eggs levels. Instead about mean EPG values, the FLOTAC gave results comparable to McMaster $(1.0 \mathrm{ml}$ ) for composite sample with low level of GI strongyle eggs, while for composite sample with high level of GI strongyle eggs, the McMaster $(0.15 \mathrm{ml})$ gave higher values.

\section{Discussion}

The results show that reliability of FECPAK and McMaster techniques for the estimation of the GI strongyle eggs EPG is influenced by the choice of the reading area (volume) as reported also by Cringoli et al. (2004) both for low and high egg counts, in fact the results are very variable between the different reading level. At the higher level of infection the smaller the area of the slides that are counted the higher will be the egg counts confirming the observation of Cringoli et al. (2004). It is probable that microcirculation in the slides is tending to centralise the eggs. If an approximate count is required in order to decide whether treatment of cattle is necessary any of the methods will be suitable. The larger variation $(\mathrm{CV})$ found with McMaster and FECPAK is due to the large multiplication factors used to provide eggs per gram from the egg counts. When used for horses allowance must be made for the uneven distribution of eggs (Presland et al. 2005) by thorough mixing of the faecal sample. However measurement of $20 \mathrm{Mc}-$ Master different slides has shown that the volume under the slide varies significantly from slide to slide and so some McMaster slides contain morethan $0.5 \mathrm{ml}$ which will increase egg counts (Cringoli et al. unpublished data).

The ability to reliably count low numbers of eggs becomes important if the resistance status of nematodes in a cattle herd is required especially when the initial egg counts are not high (below 100 EPG). Two sensitive methods not tested here are the Wisconsin flotation method (Cox and Todd, 1962; Egwang and Slocombe, 1982) and the modified salt-sugar flotation method (Mes, 2003). The Wisconsin method requires centrifugation as does FLOTAC. This need to centrifuge has now been overcome with Mini-FLOTAC (Cringoli et al. 2013), sensitive to $5 \mathrm{EPG}$, but which maintains the advantage of FLOTAC of translation (moving the layer of salt with the eggs out of the light path that containing most of the debris) making the slides much easier to read. If combined with another invention, Fill-FLOTAC which contains a built in measuring cone and filter for sample preparation, the need to weigh samples is removed making on farm counting of nematode eggs very easy. Accurate counts with minimum work must become important as veterinary surgeons encourage all farmers to know the resistance status of nematodes in cattle and in other grazing animals on their farms. However, more comparison studies still need to be sound in order to promote this new technique for the parasitological diagnosis in cattle.

\section{References}

Coles G.C., Bauer C., Borgsteede F.H., Geerts S., Klei T.R., Taylor M.A., Waller P.J. 1992. World association for the advancement of veterinary parasitology (W.A.A.V.P.) methods for the detection of anthelmintic resistance in nematodes of veterinary importance. Veterinary Parasitology, 44, 35-44.

Cox D.D., Todd A.C. 1962. Survey of gastrointestinal parasitism in Wisconsin dairy cattle. Journal of the American Veterinary Medical Association, 141, 706-709.

Cringoli G., Rinaldi L., Veneziano V., Capelli G., Scala A. 2004. The influence of flotation solution, sample dilution and the choice of McMaster slide area (volume) on the reliability of the McMaster technique in estimating the faecal egg counts of gastrointestinal strongyles and Dicrocoelium dendriticum in sheep. Veterinary Parasitology, 123, 121-131.

Cringoli G., Rinaldi L., Maurelli M.P., Utzinger J. 2010. FLOTAC: new multivalent techniques for qualitative and quantitative copromicroscopici diagnosis of parasites in animals and humans. Nature Protocols, 5, 503-551. DOI: 10.1038/nprot. 2009.235 .

Cringoli G., Rinaldi L., Albonico M., Bergquist R., Utzinger J. 2103. Geospatial(s) tools: integration of advanced epidemiological sampling and novel diagnostics. Geospatial Health, 7, 399 404.

Egwang T.G., Slocombe J.O. 1982. Evaluation of the Cornell-Wisconsin centrifugal flotation technique for recoverins trichostrongylid eggs from bovine feces. Canadian Journal of Comparative Medicine, 46, 133-137.

Gordon H., Whitlock H.V. 1939. A new technique for counting nematode eggs in sheep faeces. Journal Council Scientific Industrial Research, 12, 50-52.

Levecke B., Rinaldi L., Charlier J., Maurelli M.P., Bosco A., Vercruysse J., Cringoli G. 2012. The bias, accuracy and precision of faecal egg count reduction test results in cattle using McMaster, Cornell-Wisconsin and FLOTAC egg counting methods. Veterinary Parasitology, 188, 194-199. DOI: 10.1016/j.vetpar.2012.03.017.

Levecke B., Rinaldi L., Charlier J., Maurelli M.P., Morgoglione M.E., Vercruysse, J., Cringoli G. 2011. Monitoring drug efficacy against gastrointestinal nematodes when faecal egg counts are low: do the analytic sensitivity and the formula matter? Parasitology Research, 109, 953-957. DOI: 10.1007/ s00436-011-2338-z 
MAFF, 1986. Manual of Veterinary Parasitological Techniques, Her Majesty's Stationary Office, London (1986) 160 pp.

Mes T.H.M. 2003. Technical variability and required sample size of helminth egg isolation procedures. Veterinary Parasitology, $115,311-320$

Presland S.L., Morgan E.R., Coles G.C. 2005. Counting nematode eggs in equine faecal samples. Veterinary Record, 156, 208-210.

Received: January 25, 2014

Revised: April 14, 2014

Accepted for publication: June 27, 2014
Sutherland I.A., Leathwick D.M. 2011. Anthelmintic resistance in nematode parasites of cattle: global issue? Trends in Parasitology, 27, 176-181. DOI: 10.1016/j.pt.2010.11.008. 